\title{
LEVANTAMENTO EPIDEMIOLÓGICO DOS CASOS DE TUBERCULOSE NOTIFICADOS NO MUNICÍPIO DE CERES-GOIÁS NO PERÍODO COMPREENDIDO ENTRE 2001 A 2012
}

\author{
SURVEY EPIDEMIOLOGICAL OF TUBERCULOSIS CASES NOTIFIED IN \\ THE CITY OF CERES-GOIAS IN THE PERIOD FROM 2001 TO 2012
}

\section{Milce Costa}

Doutora em Medicina Tropical. Docente da FACER - Faculdades. Ceres - GO, Brasil. milcebiomol@yahoo.com.br

\section{Ana Cláudia Lacerda}

Acadêmica do curso de Farmácia, FACER - Faculdades. Ceres - GO, Brasil.

anaclaudia.lacerda@hotmail.com

\section{Heloíza Dias Lopes Lago}

Especialista em Saúde Pública e em Enfermagem em Unidade de Terapia Intensiva. Docente da FACER - Faculdades. Ceres - GO, Brasil.

heloizalago@hotmail.com

\section{Andréia Cristina da Silva}

Graduada em Farmácia pela FACER - Faculdades. Ceres - GO, Brasil.

andreia.farmacia2008@gmail.com

\section{Daniela de Jesus Campos}

Graduada em Farmácia pela FACER - Faculdades. Ceres - GO, Brasil.

\section{Suelem Kácia Bueno}

Graduada em Farmácia pela FACER - Faculdades. Ceres - GO, Brasil.

\section{RESUMO}

Introdução: A tuberculose (TB) é uma doença infecciosa crônica conhecida mundialmente como um problema de saúde pública de grande proporção que aflige a humanidade desde a antiguidade. Metodologia: Trata-se de um estudo epidemiológico de levantamento de dados de aspecto quantitativo, onde foi realizada uma investigação epidemiológica dos casos de TB no município de Ceres-GO no período compreendido entre 2001 a 2012 através do TABWIN/SINANET. Resultados e Discussão: De acordo com o banco de dados, o município de Ceres apresentou 58 casos de TB por M. tuberculosis. A forma clínica da TB que mais acometeu a população foi a forma pulmonar com 49 (84,5\%) dos casos, provavelmente, isso ocorra devido a principal via de infecção da doença ser por via aérea. $\mathrm{O}$ gênero masculino foi predominante em $44(75,9 \%)$ dos casos, certamente em virtude de hábitos comportamentais que tornam este grupo mais exposto à doença, como o etilismo e o tabagismo. Com relação à faixa etária, pode-se visualizar o predomínio dos indivíduos entre 40 a 59 anos com $21(36,2 \%)$ dos casos notificados, acredita-se que este resultado esteja relacionado à eficácia da vacina $\mathrm{BCG}$ e principalmente pelo aumento dos casos de infecção com HIV/AIDS. Dos 58 casos notificados em Ceres-GO $32(55,1 \%)$ dos casos de TB 
obtiveram a cura. Conclusão: Portanto, mesmo com o desenvolvimento estratégias para prevenção e controle da TB, o número de notificações ainda exige preocupação constante dos setores responsáveis. Dessa forma, deve-se continuar investindo rigorosamente nas medidas preventivas usuais e eficazes contra a TB como a vacinação e a quimioprofilaxia.

Palavras chave: Tuberculose. Epidemiologia. Incidência. Forma clínica. Mortalidade.

\begin{abstract}
Introduction: Tuberculosis (TB) is an infectious chronic disease known the world over as a public health problem of great proportions for many centuries. Methodology: An epidemiological study employing a quantitative aspect of evaluation of data was carried out in which an investigation of cases of TB in the municipality of Ceres, Goiás, was effected for the period from 2001 to 2012, utilizing the TABWIN/SINANNET. Results and discussion: According to the data bank, Ceres presented 58 cases of TB M.tuberculosis. The clinical form of TB which most afflicted the population was the pulmonary type with 49 (84.5\%) of the cases; probably this occurred because the principal means of infection from this is through the air. The masculine gender was predominant in $44(75.9 \%)$ of the cases, certainly in virtue of comportmental habits such as alcoholism and tobacco addiction, which lead this group to more exposure to the disease. With respect to age level, it may be seen that the majority of the individuals were between 40 and 59 years of age with 21 (36.2\%) of the cases reported; it is believed that this figure is related to the efficacy of the BCG vaccine and principally to the increase in cases of infection by HIV/AIDS. Of the 58 reported cases in Ceres, Goias, 32 (55.1\%) of the cases of TB were cured. Conclusion: Therefore, even with the development of strategies for the prevention and control of $\mathrm{TB}$, the number of reported cases demands constant concern from the responsible sectors. In this manner, rigorous investment should be continued in the usual and effective preventive measures against TB, such as vaccination and chemical intervention.
\end{abstract}

Key words: Tuberculosis. Epidemiology. Incidence. Clinical form. Mortality.

\title{
Endereço para correspondência:
}

Av. Brasil, S/N, Qd. 13 Morada Verde Ceres - Go, Fone: (62) 3323-1040,

E-mail: milcebiomol@yahoo.com.br

\section{INTRODUÇÃO}

A tuberculose (TB) é uma doença infecciosa crônica reconhecida mundialmente como um problema de saúde pública de grande proporção que aflige a humanidade desde a antiguidade. Na atualidade, a TB continua a exigir preocupação constante das autoridades sanitárias de todo o mundo, principalmente devido à sua crescente incidência em diferentes grupos populacionais mais susceptíveis à doença como imunocomprometidos, moradores de rua e indivíduos que vivem em ambientes restritos de liberdade, entre outros (MASCARENHAS et al., 2005). 
O agente etiológico da TB é uma micobactéria pertencente ao gênero Mycobacterium da família Mycobacteriaceae. Morfologicamente, o micro-organismo se caracteriza por ser um bacilo reto ou ligeiramente curvo, cuja dimensão varia de 0,2 a 0,6 micrômetros por 1 a 10 micrômetros, imóvel, não esporulado, não encapsulado, não produz toxinas e é capaz de sobreviver no interior de células fagocitárias, o que o caracteriza como um patógeno intracelular aeróbio estrito (LORENZ et al., 2009; MELO et al., 2009). A via de infecção tuberculosa é, quase sempre, inalatória, dessa forma, o Mycobacterium tuberculosis apresenta uma maior predileção pelos pulmões, sendo assim, a tuberculose pulmonar se apresenta como a forma clínica mais encontrada nos indivíduos infectados (FERREIRA et al., 2005; SOUZA, 2009).

Com o surgimento da síndrome da imunodeficiência adquirida (SIDA) a TB tem sido considerada uma das doenças mais frequentes associadas à infecção pelo HIV/VIH (human Immunodeficiency virus/vírus da imunodeficiência humana) em razão da maior virulência do M. tuberculosis. O HIV é responsável não apenas pelo crescimento do número de casos de TB, mas também tem contribuído significativamente no aumento da mortalidade entre os pacientes co-infectados. A co-infecção HIV/TB também aumentou as dificuldades em relação ao controle da TB, tanto nos países desenvolvidos quanto naqueles em desenvolvimento (MASCARENHAS et al., 2005; NUNN et al., 2005; NEVES et al., 2010).

Segundo estimativas da OMS (Organização Mundial de Saúde) o Brasil com outros 22 países somam $80 \%$ dos casos mundiais de TB. Na América Latina, Brasil e Peru totalizam $50 \%$ dos casos estimados e somando-se Bolívia, Equador, Haiti, Honduras, Guiana, México, Nicarágua e República Dominicana, chega-se a 75\% dos casos estimados no mundo (HIJJAR et al., 2005; COUTINHO et al., 2012). Segundo dados do Ministério da Saúde são observados que no Brasil mais de 50 milhões de pessoas podem encontrar-se infectadas pelo M. tuberculosis com aproximadamente 80 mil casos novos por ano e cerca de 5 mil mortes causadas pela doença (BRASIL, 2009). No ano de 2007 foram notificados no Brasil 72.194 casos novos de TB, demonstrando um coeficiente de incidência de 38/100.000 hab. Destes, 41.117 casos novos foram bacilíferos (baciloscopia de escarro positiva), apresentando um coeficiente de incidência de 41/100.000 hab. Estes números colocam o Brasil na $19^{a}$ posição em relação ao número de casos de TB no mundo e na $104^{\circ}$ posição em relação ao coeficiente de incidência (BRASIL, 2010d). No Brasil, no ano de 2011, os estados do Amazonas e Rio de Janeiro foram os que mostraram as maiores taxas de incidência de TB no país, 62,6\% e 57,6\% 
respectivamente, enquanto os estados de Goiás e Distrito Federal foram os que apresentaram as menores taxas da doença, 13,6\% e 11,1\% respectivamente (BRASIL, 2012).

Uma vez que o Brasil figura como um dos países com elevada incidência da doença, implementou-se no país através do Ministério da Saúde o Programa Nacional de Controle da Tuberculose (PNCT). Este programa visa principalmente ações de prevenção, vigilância e controle da TB. Pelo programa objetiva-se reduzir as fontes de infecção utilizando-se de estratégias relacionadas à descentralização e horizontalização das ações de prevenção principalmente no campo dos cuidados primários de saúde, incluindo-se o diagnóstico clínico e laboratorial da doença, bem como o tratamento. O tratamento por sua vez, é realizado gratuitamente a todos os pacientes que estejam cadastrados e acompanhados nas Unidades Básicas de Sáude (UBS), consequentemente reduzindo a incidência, prevalência e mortalidade causada pela TB (HIJJAR et al., 2005; BARREIRA; GRANGEIRO, 2007).

Apesar de se conhecer seu agente etiológico, sua forma de transmissão e de se dispor de tratamento eficaz, a TB ainda continua preocupando as autoridades sanitárias até os dias de hoje, principalmente devido ao aumento dos casos de AIDS, o que torna o portador mais suscetível à infecção pelo $M$. tuberculosis. Em virtude da insuficiência de dados científicos disponíveis na literatura científica sobre TB na microrregião de Ceres-GO, este estudo realizou um levantamento epidemiológico dos casos notificados de TB neste município no período compreendido entre 2001 a 2012.

\section{METODOLOGIA}

\section{Tipo de Estudo}

Trata-se de um estudo transversal, analítico, com abordagem quantitativa, onde foi realizada uma investigação epidemiológica dos casos de TB no município de Ceres - GO no período compreendido entre 2001 a 2012.

\section{Caracterização do município}

A cidade de Ceres - GO possui 20.722 habitantes numa área de aproximadamente 214 quilômetros quadrados, conforme contagem populacional do IBGE (Instituto Brasileiro de Geografia e Estatística). Somando-se a população dos 05 municípios limítrofes (Rialma, Carmo do Rio Verde, Nova Glória, Rubiataba, Ipiranga de Goiás) totaliza-se 70.440 habitantes na região (IBGE, 2010a). 


\section{População de estudo}

A população de estudo consistiu em todos os casos de TB que foram notificados no Sistema de Informação de Agravos de Notificação (TABWIN/SINANNET) referentes à população do município de Ceres- GO.

\section{Fonte de Dados}

Os dados de notificação da ocorrência de TB na população do município de Ceres-GO foram obtidos na Secretaria de Saúde do município através do TABWIN/SINANET (Sistema local do município). Quanto à taxa de mortalidade por TB, foi utilizado o Sistema TABNET/DATASUS disponível no site do Ministério da Saúde. Dados sobre o censo populacional referente aos residentes no município de Ceres-GO foram obtidos no site do Instituto Brasileiro de Geografia e Estatística (IBGE 2010).

\section{Variáveis}

Foram analisadas no estudo: variáveis epidemiológicas (gênero e faixa etária), variáveis relacionadas aos aspectos clínicos (forma clínica da TB e mortalidade) e variável cronológica (período analisado).

\section{Análise de dados}

A análise foi realizada nos bancos de dados disponibilizados TABWIN/SINANNET e TABNET/DATASUS no período de 2001 a 2012. Após a obtenção dos valores absolutos dos casos de TB notificados e confirmados, os dados foram organizados em planilha do programa Microsoft巴 Excel onde foram analisadas as variáveis epidemiológicas e os aspectos clínicos da TB no município de Ceres-GO. Posteriormente foram feitas análise e interpretação dos resultados.

\section{RESULTADOS E DISCUSSÃO}

Este estudo avaliou a situação epidemiológica da TB no Município de Ceres - GO através do banco de dados TABWIN/SINANNET. De acordo com o banco de dados, o município de Ceres - GO apresentou 58 casos de TB notificados no período de 2001 a 2012. 
De acordo com os dados obtidos no TABWIN/SINANNET verificou-se que no município de Ceres-GO, nos anos de 2001 a 2012, a forma clínica da TB que mais acometeu a população foi a forma pulmonar com 49 (84,5\%) dos casos. A forma extrapulmonar é verificada em $8(13,8 \%)$ dos casos e a forma pulmonar e extrapulmonar concomitantemente figura em $1(1,7 \%)$ dos casos descritos (Figura 1).

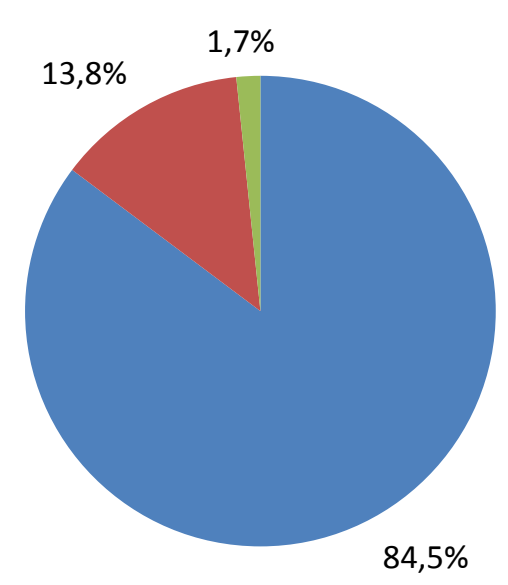

- Pulmonar

- Extrapulmonar

Extra + Pulmonar

Figura 1. Casos notificados de TB no município de Ceres-GO segundo a forma clínica no período compreendido entre 2001 a 2012.

Adaptado: Secretaria Municipal de Saúde. TABWIN/SINANNET

Os dados obtidos a respeito das formas clínicas da TB verificadas no município de Ceres - GO apresentam concordância com estudos realizados em outros estados em nosso país. No Rio de Janeiro, Hijjar et al. (2005), relatam que 85,2\% das pessoas que são acometidas por TB apresentam a forma clínica pulmonar e apenas 14,8\% apresentam as formas extrapulmonares. No estudo de Seiscento et al. (2009), realizado no Estado de São Paulo, verificou-se situação semelhante, apontando que a principal forma clínica da TB encontrada foi a pulmonar com $82,2 \%$ dos casos, no entanto, o percentual para as formas extrapulmonares neste estudo foi de $17,8 \%$.

$\mathrm{O}$ fato da forma pulmonar da TB apresentar maior predominância sobre a extrapulmonar provavelmente ocorra devido a principal via de infecção da doença ocorrer por via aérea, pelo contato direto pessoa/pessoa através da inalação de partículas carregadas de bacilos aerolizadas pelo indivíduo doente. Embora os bacilos possam se instalar em outros locais no organismo, sua forte afinidade pelo oxigênio faz com que estes tenham predileção por órgãos como os pulmões, que apresentam alta concentração de oxigênio. Dessa forma, esses locais tornam-se preferenciais para a instalação, proliferação e transmissão do $M$. 
tuberculosis, uma vez que a bactéria é um aeróbio estrito (MASCARENHAS et al., 2005; CALIARI, 2009; BRASIL, 2011).

O gênero predominante mais acometido pela TB em Ceres-GO no período de 2001 a 2012 foi o masculino, verificado em 44 (75, 9\%) dos casos. O gênero feminino, portanto, apresenta $14(24,1 \%)$ dos casos notificados (Figura 2).

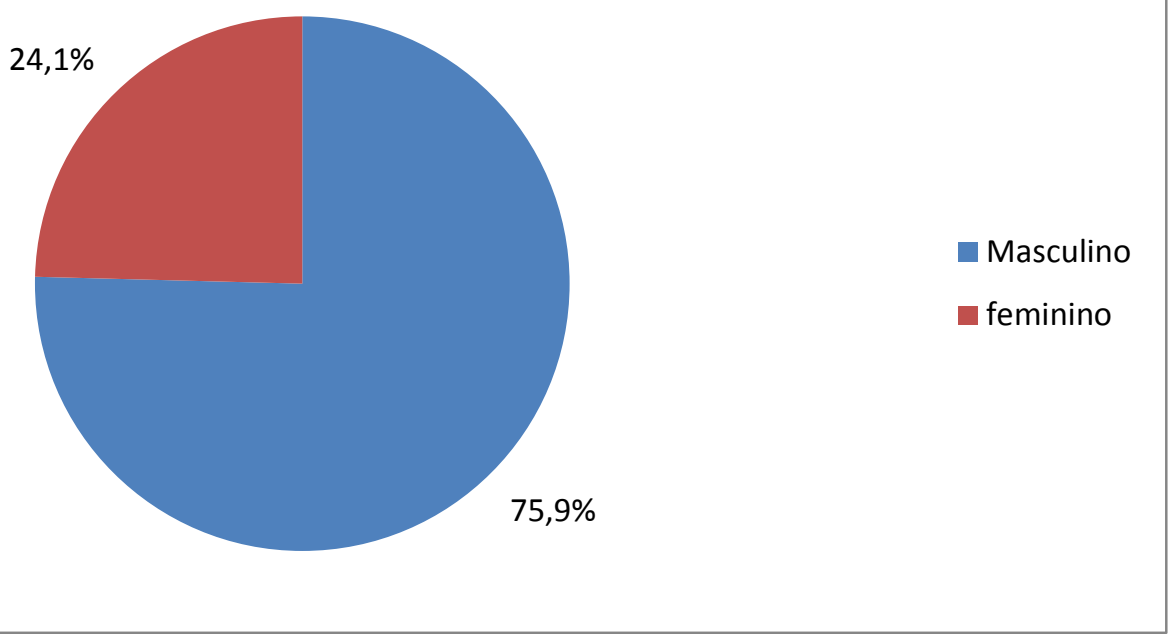

Figura 2. Casos notificados de TB no Município de Ceres-GO segundo o gênero dos pacientes no período compreendido entre 2001 a 2012.

Fonte: TABWIN/SINANNET

A predominância de TB em pacientes do gênero masculino também foi observada no estudo de Seiscento et al. (2009), no Estado de São Paulo, onde verificou-se a proporção de 2:1 em relação ao gênero feminino. Na pesquisa desenvolvida por Otsuka (2008), existem relatos do predomínio de casos positivos $(70,8 \%)$ para TB em pacientes do gênero masculino. Zocche e Silva (2009), em seu estudo, apontaram que de todos os casos de TB notificados, $72,8 \%$ pertenciam ao gênero masculino e apenas $26,8 \%$ dos casos ao gênero feminino.

De acordo com Santo et al. (2009), e Otsuka (2008), a situação da população acometida pela TB ser predominantemente masculina, provavelmente se deve ao fato de ser o grupo mais exposto à doença em virtude de hábitos comportamentais como o etilismo e o tabagismo, e a demora na procura do serviço de saúde. O etilismo frequente pode ser considerado um importante fator de risco para o desenvolvimento da TB, uma vez que o indivíduo deixa de se alimentar bem, podendo apresentar um quadro de desnutrição, diminuindo assim, sua imunidade e tornando-se mais suscetível à doença (ANDRADE et al., 2005; PAIVA et al., 2011). 
Em relação ao tabagismo, a exposição do indivíduo à fumaça do tabaco, passiva ou ativamente, também é considerada um fator de risco para a TB. Os princípios fisiopatológicos nos quais o tabagismo aumenta o risco de adquirir tuberculose podem ser explicados pela disfunção da mecânica ciliar, que aumenta a aderência das bactérias no trato respiratório superior, diminuição da resposta imune do paciente, defeitos na resposta imunológica dos macrófagos e diminuição dos níveis de CD4+, aumentando a suscetibilidade à infecção pelo M. tuberculosis. Além disso, o tabagismo ainda reduz a concentração de oxigênio no sangue. Todos estes fatores irão colaborar para a gravidade das lesões necrotizantes pulmonares, tornando mais lenta a cicatrização, o que pode gerar sequelas mais extensas (BRASIL, 2010b; RABAHI, 2012).

Socialmente, verifica-se que os homens normalmente procuram os serviços de saúde em virtude de doenças e não como forma de prevenção. Especificamente na TB, os homens atrasaram mais que as mulheres para buscar por atendimento nos serviços de saúde (WYSOCKI et al., 2013).

Com relação à faixa etária mais acometida por TB no município de Ceres-GO no período de 2001 a 2012, pode-se verificar o predomínio dos indivíduos entre 40 a 59 anos com $21(36,2 \%)$ dos casos notificados no TABWIN/SINANNET (Figura 3).

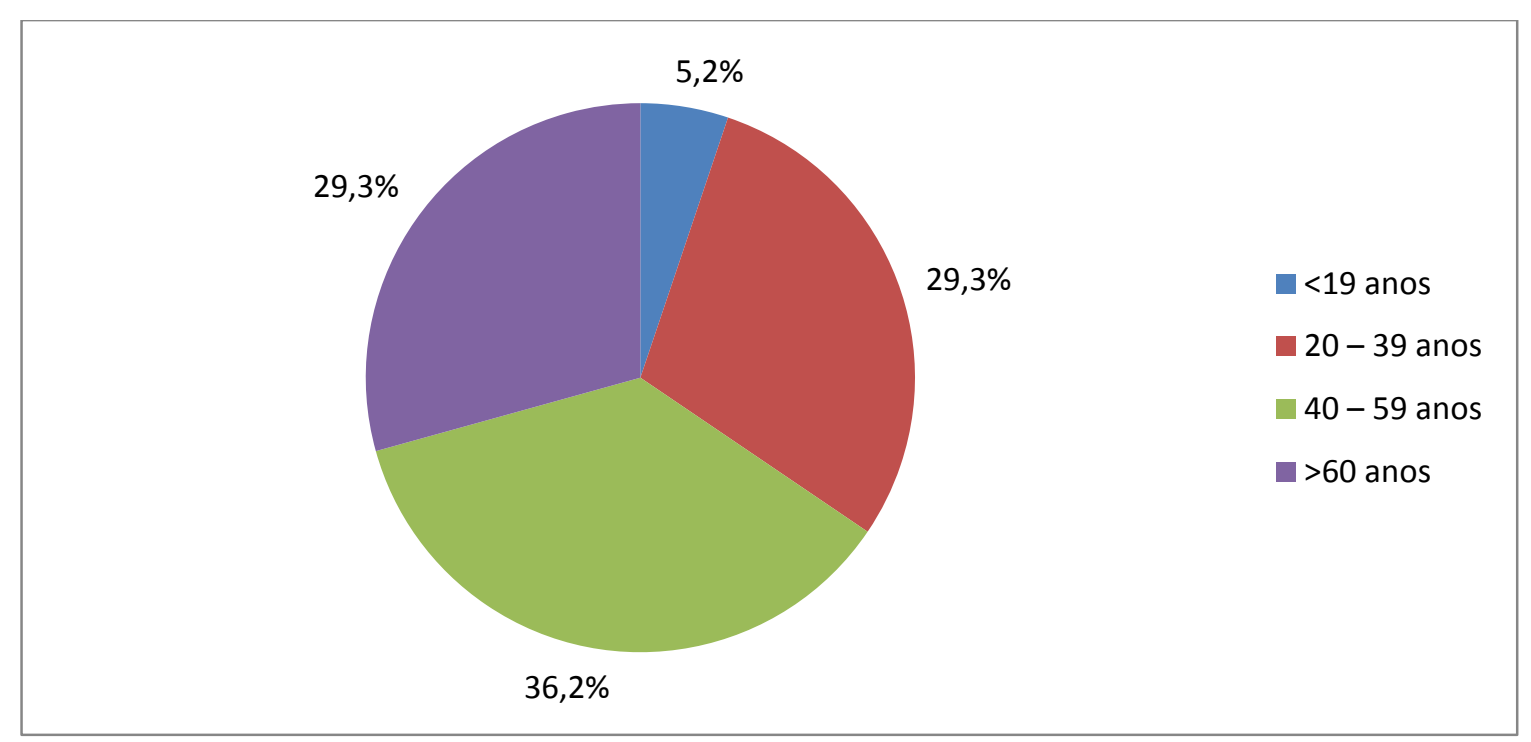

Figura 3. Casos notificados de TB no município de Ceres-GO segundo a faixa etária no período compreendido entre 2001 a 2012.

Fonte: TABWIN/SINANNET

Outros autores também observaram percentuais de TB semelhantes nesta faixa etária. Okaruma (2003) em São Paulo, Rodrigues et al. (2010) no Rio Grande do Sul e Vieira et al. 
(2011) em Mossoró-RN, verificaram incidências de 31,3\%, 32,2\% e 33,3\% de TB respectivamente nos pacientes entre 40-59 anos pesquisados. Acredita-se que a característica etária da população adulta ser mais acometida pelo $M$. tuberculosis esteja relacionada, por um lado, pela eficácia da vacina BCG, que diminui o risco da infecção na população mais jovem, e, por outro, pelo crescimento da população de adultos e idosos mais susceptíveis ao bacilo, principalmente considerando-se a tendência do aumento dos casos de infecção com HIV/AIDS, na qual a TB entra como uma co-infecção muito prevalente (SANTO et al., 2009).

Quanto à situação atual dos casos de TB notificados, o Ministério da Saúde padroniza informações através do TABWIN/SINANNET, utilizando a terminologia "Situação encerrada" para designar: casos ignorados/branco, casos de cura; abandono do tratamento; óbito por outras causas, transferência e mudança de diagnóstico. Dos 58 casos notificados em Ceres-GO, $32(55,1 \%)$ dos casos de TB obtiveram a cura da doença, $10(17,2 \%)$ dos pacientes foram transferidos para continuidade do tratamento em outra Unidade de Saúde e 08 (14\%) dos indivíduos abandonaram o tratamento (Figura 4).

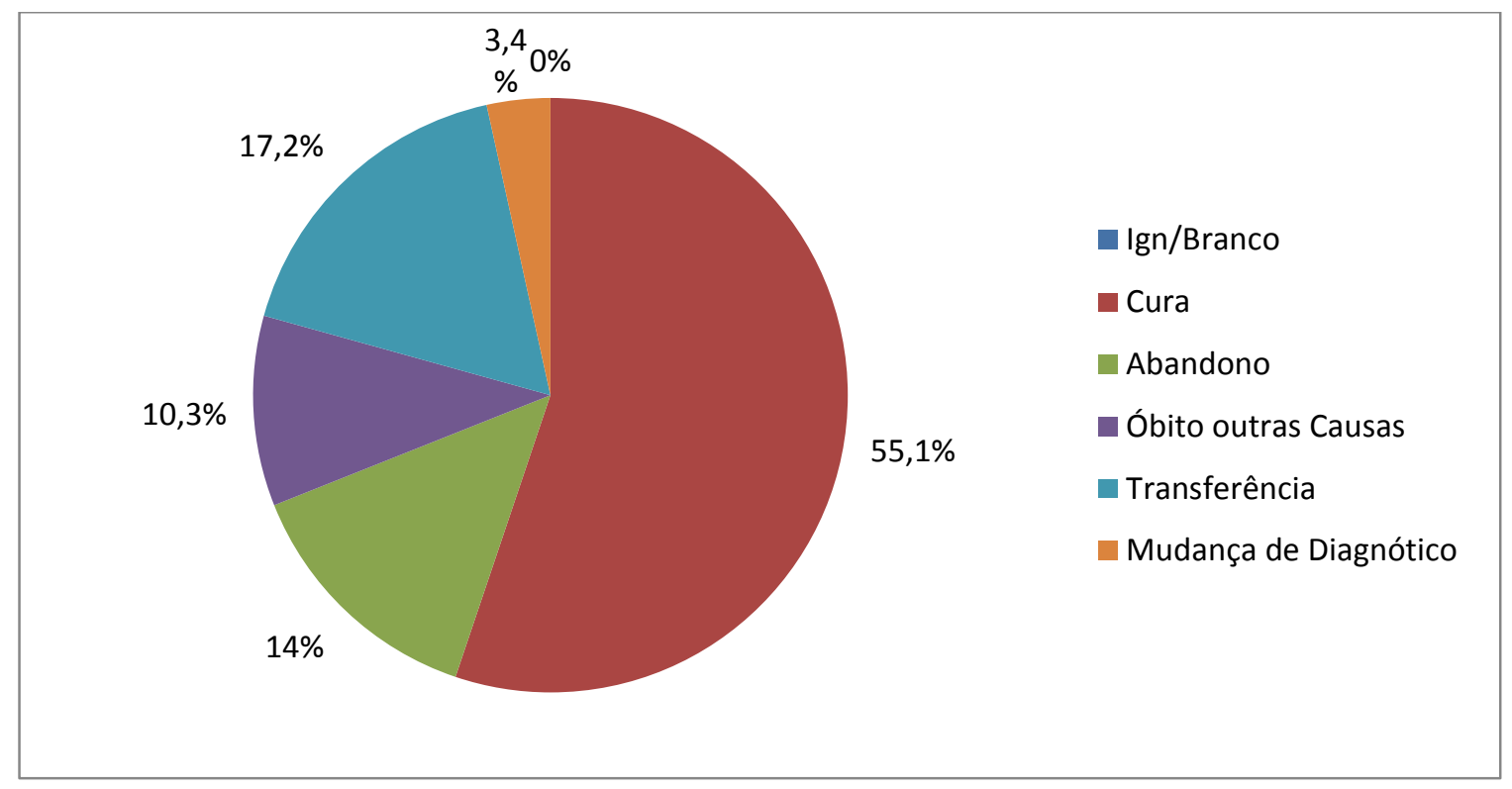

Figura 4. Situação encerrada dos casos notificados de TB no Município de Ceres-GO no período compreendido entre 2001 a 2012.

Fonte: TABWIN/SINANNET

Durante os onze anos avaliados neste estudo, 32 (55,1\%) dos pacientes notificados no município de Ceres-GO obtiveram a cura da TB, no entanto, este percentual não atinge a meta de cura dos pacientes tuberculosos segundo o preconizado pelo PNCT. Neste contexto é 
importante ressaltar que uma das prioridades do Programa é que os estados brasileiros atinjam a meta global com $85 \%$ de cura dos doentes detectados e diagnosticados por TB e a taxa de abandono seja inferior à 5\% (BRASIL, 2010c). O percentual de 55\% certamente pode ser explicado pelo considerável percentual de abandono do tratamento (14\%), maior do que o recomendado pelo PNCT e também pela transferência dos pacientes para outras unidades de saúde em outro município (17,2\%). Por ser um tratamento longo, o paciente passa a se sentir melhor nos primeiros meses e alguns se consideram curados antes da cura efetiva e abandonam a terapia medicamentosa. Em outros casos, os efeitos colaterais dos medicamentos usados como: intolerância gástrica, manifestações cutâneas e artralgia, contribuem para a interrupção do tratamento (SÁ et al., 2007; GIROTI et al., 2010). Outro fator para o não cumprimento dessa meta é pelo fato de que muitos pacientes diagnosticados em determinado município, são transferidos para dar continuidade ao tratamento nas cidades onde residem, sem que a evolução da doença seja acompanhada na unidade de saúde onde se iniciou o tratamento (COÊLHO et al., 2010).

Ao analisar os dados sobre a mortalidade por TB no Município de Ceres-GO, observou-se que ocorreram 04 óbitos $(6,9 \%)$ no período pesquisado. Destes, todos os indivíduos $(100 \%)$ pertenciam ao gênero masculino. Diversos estudos mostram que a taxa de mortalidade por TB é predominantemente maior no gênero masculino em relação ao feminino acompanhando o padrão de incidência da doença nesse gênero (COÊLHO et al., 2010, MACIEL et al., 2011, SOUSA, PINHEIRO 2011, SOUSA et al., 2012). No entanto, Maciel et al., 2011, em trabalho realizado no estado de Pernambuco, no município de Salgueiro-PE descrevem que $100 \%$ dos óbitos ocorreram em homens, corroborando desta forma com este estudo. Alguns fatores podem ser relevantes para mortalidade masculina, como a procura do serviço de saúde mais tardiamente, ao abandono do tratamento, especialmente por homens jovens solteiros e separados, que aderem menos às recomendações médicas e não abandonam hábitos prejudiciais ao tratamento, como o etilismo e tabagismo, uma vez que estes podem influenciar no prognóstico da doença (MENDES; FENSTERSEIFER, 2004; HINO et al., 2007; MOREIRA et al., 2008; PAIVA et al., 2011).

\section{CONCLUSÃO}

A pesquisa permitiu verificar os casos de tuberculose notificados no município de Ceres-GO entre 2001 a 2012. Observou-se que a forma clínica da TB que mais acometeu a população foi a pulmonar, que maioria dos indivíduos com a doença pertenciam ao gênero 
masculino, que a faixa etária mais frequente foi entre 40 a 59 anos e que o percentual de cura de $55,1 \%$ não atinge a meta de cura dos pacientes tuberculosos segundo o preconizado pelo PNCT, que é de $85 \%$. Ou seja, mesmo com o desenvolvimento estratégias para prevenção e controle da TB disponíveis no país, o número de notificações ainda exige preocupação constante dos setores responsáveis. Portanto, diante dos dados obtidos nesta pesquisa é de fundamental importância que se continue investindo rigorosamente nas medidas preventivas usuais e eficazes contra a TB como a vacinação, amplamente divulgada em campanhas nacionais e a quimioprofilaxia, padronizada pelo PNCT em todo o país e executadas pelos estados e municípios. Vale ressaltar que medidas educativas através de palestras para conscientizar o doente e sua família, podem facilitar a adesão e a conclusão do tratamento desses pacientes com probabilidade de sucesso, principalmente alcançando a meta recomendada pelo $\mathrm{PNCT}$.

\section{REFERÊNCIAS}

ANDRADE, R.L.P. et al. A influência do alcoolismo no prognóstico e tratamento da tuberculose. Rev. Eletrônica Saúde Mental, Álcool e Drogas. Ribeirão Preto, v.1, n.1, p.1-9, 2005.

BARREIRA, D. GRANGEIRO, A. Avaliação das estratégias de controle da tuberculose no Brasil. Rev Saúde Pública. 41(Supl. 1), 2007.

BRASIL. Secretaria de Vigilância em Saúde. Boletim Epidemiológico. Especial Tuberculose. 2012, v.43. 12p. Disponível em: $\langle$ http://portal.saude.gov.br/portal/arquivos/pdf/bolepi_v43_especial_tb_correto.pdf > Acesso em 17/10/2014, 09:20 horas

BRASIL, Ministério da Saúde. Jovem/ Mulher e Tabaco. Brasil, 2011. São Paulo. Disponível em: 〈http://www1.inca.gov.br/tabagismo $>$. Acesso em 24/10/2014, 15:25 horas

BRASIL. Instituto Brasileiro de Geografia e Estatística. Brasil, 2010a. Disponível em: <http://www.ibge.gov.br/estadosat/perfil.php?sigla=go> Acesso em 03/11/2014, 16:40 horas.

BRASIL. Ministério da Saúde. Secretaria de Vigilância em Saúde. Programa Nacional de Controle da Tuberculose. Manual de Recomendações para o Controle da Tuberculose no Brasil. [s.1.], 2010b. 186p. 
BRASIL. Ministério da Saúde. Secretaria de Vigilância em Saúde. Departamento de Vigilância Epidemiológica. Programa Nacional de Controle da Tuberculose. Nota técnica sobre as mudanças no tratamento da tuberculose no Brasil para adultos e adolescentes. [s.1.], 2010c. 2 p.

BRASL. Ministério da Saúde. Sistema Nacional de Vigilância em Saúde. Relatório de Situação: Goiás. Brasília, 2010d. 62 p.

BRASIL. Ministério da Saúde. Secretaria de Vigilância em Saúde. Departamento de Vigilância Epidemiológica. Doenças infecciosas e parasitarias: Guia de Bolso. $7^{\mathrm{a}}$ edição. CID 10: A15 a A19. Caderno 7. Brasília, 2009.

CALIARI, J.S. Perfil dos doentes de Tuberculose nos anos de 2007-2008 no departamento da regional de saúde III no Estado de São Paulo. 87f. Dissertação (Pós Graduação em Enfermagem) - Universidade Federal de São Carlos - SP. São Carlos - SP, 2009.

COÊLHO, D.M.M. et al. Perfil epidemiológico da tuberculose no Município de Teresina-PI, no período de 1999 a 2005. Epidemiol. Serv. Saúde. Brasília, v.19, n.1, 2010.

COUTINHO, L.A.S.A.; OLIVEIRA, D.S.; SOUZA, G.F.; FERNANDES FILHO, G.M.C.; SARAIVA, M.G. Perfil Epidemiológico da Tuberculose no Município de João Pessoa - PB, entre 2007 - 2010. Revista Brasileira de Ciências da Saúde, São Caetano do Sul, v.16, n. 1, pp.35-42, 2012.

FERREIRA, A.A.A. et al. Os fatores associados à tuberculose pulmonar e a baciloscopia: uma contribuição ao diagnóstico nos serviços de saúde pública. Rev Bras Epidemiol. 8(2): 142-9, 2005.

GIROTI, S.K.O. et al. Perfil dos pacientes com tuberculose e os fatores associados ao abandono do tratamento. Cogitare Enferm. [s.1.], v.15, n.2, p.271-277, 2010.

HIJJAR, M.A. et al. Epidemiologia da tuberculose: importância no mundo, no Brasil e no Rio de Janeiro. Rev. Pulmão. Rio de Janeiro, v.14, n.4, p.310-314, 2005.

HINO, P. et al. Série histórica da mortalidade por tuberculose no Brasil (1980-2001). Rev. Latino-am Enfermagem. [s.1.], v.15, n.5, p.53-58, 2007

LORENZ, C.G. et al. Pesquisa de BAAR por meio de Modificações na Coloração de ZiehlNeelsen e por Gram. LAES \& HAES. [s.1.], n.180, p. 178-186, 2009. 
MACIEL, S.S.S.V. et al. Internação hospitalar por tuberculose respiratória nas gerências regionais de saúde de Pernambuco. Revista da AMRIGS. Porto Alegre, 55 (3): 229-233, jul.set. 2011

MASCARENHAS, M.D.M. et al. Perfil epidemiológico da tuberculose entre casos notificados no Município de Piripiri, Estado do Piauí, Brasil. Epidemiol. Serv. Saúde. Brasília, v.14, n.1, 2005.

MELO, F.A.F. et al. Tuberculose. In: VERONESI, R.F. et al. Tratado de Infectologia. 4 ed. V. 1. São Paulo: Editora Atheneu. 2009. Capítulo 68, p.1263-1333.

MENDES, A.M; FENSTERSEIFER, L.M. Tuberculose: porque os pacientes abandonam o tratamento? Boletim de Pneumologia Sanitária. Rio de Janeiro, v.12, n. 1, 2004.

MOREIRA, C.M.M. et al. Mortalidade específica por tuberculose no Estado do Espírito Santo, no período de 1985 a 2004. J. Bras. Pneumol. São Paulo, v.34, n.8, 2008.

NEVES, L.A.S. et al. Adesão ao tratamento por individuos com co-infecção HIV/tuberculose: revisão integrativa da literatura. Rev Esc Enfer USP. São Paulo, v.44 .p.1135-41, 2010.

NUNN, P. et al. Tuberculosis control in the era of HIV. Nat Rev Immunol, v.10, p. 819$826,2005$.

OKARUMA, M.N. Perfil epidemiológico dos pacientes com tuberculose atendidos em um hospital geral universitário, 1999-2001. São Paulo, 2003. 122f. Dissertação (Mestre em Saúde Pública) - Faculdade de Saúde Pública da Universidade de São Paulo.

OTSUKA, A. Determinação da Faixa Etária com maior Incidência de Tuberculose em Sorocaba/SP nos anos de 2004 e 2005. Rev. Eletrônica de Biologia. [s.1.], v.1, n.1, p.62-76, 2008 .

PAIVA, V.S. et al. Perfil epidemiológico da tuberculose pulmonar em Unidade Sanitária de referência em Porto Alegre, RS. Rev. da AMRIGS. Porto Alegre, v.55, n.2, abr/jun, p.113$117,2011$.

RABAHI, M.F. Tuberculose e Tabagismo. Rev. Pulmão. Rio de Janeiro, v.21, n.1, p.46-49, 2012. 
RODRIGUES, J.L.C. et al. Prevalência de confecção tuberculose/HIV em pacientes do Centro de Saúde Modelo de Porto Alegre, Rio Grande do Sul. Scientia Medica. Porto Alegre, v.20, n.3, p.212-217, 2010.

SÁ, L.D. et al. Tratamento da tuberculose em unidades de saúde da família: histórias de abandono. Texto Contexto Enferm. Florianópolis, v.16, n.4, p.712-718, out/dez, 2007.

SANTO, L.A.L.A. et al. Perfil clínico, epidemiológico e laboratorial dos pacientes com tuberculose em hospital universitário da região do Vale do Paraíba, Estado de São Paulo. Bepa. [s.1.], v.6, n.68, p.14-21, 2009.

SEISCENTO, M. et al. Aspectos epidemiológicos da tuberculose pleural no Estado de São Paulo (1998-2005). J. Bras. Pneumol. [s.1.], v.35, n.6, p.548-554, 2009.

SOUSA, M.G.G. et al. Investigação de óbitos por tuberculose, ocorridos na Região Metropolitana do Recife (PE), registrados no Sistema de Informação de Mortalidade, entre 2001 e 2008. Cad. Saúde Colet. Rio de Janeiro, 20 (2): 153-60, 2012.

SOUSA, L.M.O; PINHEIRO, R.S. Óbitos e internações por tuberculose não notificados no município do Rio de Janeiro. Rev. Saúde Pública vol.45 no.1 São Paulo Feb, 2011.

SOUZA, M.V.N. A tuberculose e o desenvolvimento de novos tuberculostáticos em FarManguinhos (FIOCRUZ) no Rio de Janeiro. Rev. Virtual Química. Rio de Janeiro, v.1, n.1, p.9-16, 2009

VIEIRA, A.N. et al. Estudo da tuberculose no município de mossoró (rn) em 2008. Rev B.S.Publica Miolo. v. 34 _ n 4.indd 879, 2011.

WYSOCKIL, A.D. et al. Atraso na procura pelo primeiro atendimento para o diagnóstico da tuberculose. Rev. esc. enferm. USP v.47 no.2 São Paulo Apr, 2013.

ZOCCHE, T.L. SILVA, R.M. Tuberculose no município de Tubarão nos anos 2000-2006. Rev. Pulmão. Rio de Janeiro, v.18, n.2, p.70-76, 2009. 